Please quote as: Oeste, S.; Söllner, M. \& Leimeister, J. M. (2014): Engineering Peer-to-Peer Learning Processes for Generating High Quality Learning Materials. In: International Conference on Collaboration and Technology (CRIWG), Santiago, Chile. 


\title{
Engineering Peer-to-Peer Learning Processes for Generating High Quality Learning Materials
}

\author{
Sarah Oeste ${ }^{1, *}$, Matthias Söllner ${ }^{1}$, Jan Marco Leimeister ${ }^{1,2}$ \\ ${ }^{1}$ Universität Kassel, Chair of Information Systems, Kassel, Germany \\ \{sarah.oeste, soellner\}@uni-kassel.de \\ ${ }^{2}$ University of St. Gallen, Institute of Information Management, St. Gallen, Switzerland \\ janmarco.leimeister@unisg.ch
}

\begin{abstract}
Organizations are facing the challenge of transferring knowledge from experienced to novice employees and are seeking for solutions that avoid the loss of knowledge with retiring experts. A possible way for overcoming this challenge is having employees develop learning materials for their novice colleagues. Based on insights from both, education and collaboration research, designing structured collaborative peer-creation-processes seems a promising approach due to several reasons. Within a peer-creation-process participants are guided to knowledge acquisition, transfer as well as documentation for others. By developing learning materials through collaboration with people at different level of knowledge, e.g., the tacit knowledge of the expert gets codified and is ready for being used by novices. Furthermore, the collaborative creation will create learning effects even among participants and should further increase their knowledge, and the quality of the learning materials. Unfortunately, little research has addressed reusable didactically driven processes of systematically documenting knowledge that can be used by others as learning material. In order to bridge this gap we identify requirements from educational and collaboration literature and conceptualize educationally driven changes in the layer model of collaboration, e.g., to consider learning objectives in the goals layer or to integrate peer review as mechanisms for quality control in the procedures layer. This paper opens up a promising field for collaboration research and provides future research directions for reusable structured peer-creation-processes with focus on learning. This research-in-progress paper closes with a conceptual framework with requirements of a collaborative peer creation process.
\end{abstract}

Keywords: Layer Model; Collaboration Engineering; Peer Creation; Peer Learning; Knowledge.

\section{Introduction}

People with different level of knowledge are working together in organizations. Besides this, knowledge leaves organizations as a consequence of demographic change. So mechanisms for a cooperative knowledge generation become important [1]. In order to perpetuate competitive capacity, it is necessary for organizations to save and to document explicit and tacit knowledge. Also research shows that collaboration of

adfa, p. 1, 2011.

() Springer-Verlag Berlin Heidelberg 2011 
people with heterogeneous knowledge can lead to a gain in productivity [2,3]. Therefore focusing on educational research is important. In this context peer learning (PL) and peer creation (PC) comprise the integration of learners in creative PL activities or paradigm changes from a learner as consumer to a producer of learning content [4]. The challenge is to codify and document explicit and tacit knowledge in a way that it is accessible for others as the basis for knowledge acquisition. This constitutes a complex and recurring task of knowledge documentation. So far, little to no research addresses structured reusable processes for such a purpose. Focusing on structured and reusable group processes, research in collaboration engineering (CE) provides useful mechanisms. However didactical elements are necessary, but not anchored in mechanisms of CE. Thus, the integration of people with heterogeneous knowledge in a collaborative process for documentation can be a purchase. We assume that combining mechanisms of educational and CE research is an appropriate approach. Consequently, the creation of a structured group collaboration process for reusable documentation of knowledge in form of high quality learning material LM needs to be addressed. We therefore propose the following research question: What requirements need to be considered in a conceptual framework for designing a peer-creation process (PCP) for developing high quality learning material (LM)? Thereby theoretical contribution of PCP research constitutes an improvement with a level 2 contribution type in form of a first framework for designing a PCP according to a design science point of view [5]. We therefore provide basics for research in PL for developing LM and basics in CE for designing collaborative group processes in section 2. In section 3, we conceptualize a framework with requirements for designing a PCP. Beginning with the discussion of the guiding idea of PCP, we provide a description of requirements and conclude with the presentation of a framework. In section 4, we describe next steps of research and end with the expected contribution.

\section{Related Work}

\subsection{Educational Basics in Peer Learning}

Following the assumption that knowledge documentation in form of LM provokes a learning process, didactical basics are necessary. A characteristic of learning is a change in behavior based on experiences [6] like conversations and discussions [7]. Thus, someone is learning on basis of its own experiences and connects this with previous knowledge. In this context PL provides a suitable approach. A group of people learn or attempt to learn something together through social interactions [8]. These interactions, like discussions with peers, foster reflection and cognitive processes [9]. That results in positive effects for the learner, called peer: e.g. knowledge gain which leads to learning success or improvement of communication skills and the peer learns to become responsible for his activities [10],[11],[7],[12]. In addition a peer learning process focuses on the learner and permits interactions between learners on the same level of knowledge [12, 13]. In most cases a lecturer prepares basic conditions and assists the process [14]. Table 1 shows the concept of PL and different kinds. PL, peer tutoring and cooperative learning provide insights on how learning processes are 
structured and conducted, but do not focus on LM development. For documenting explicit and tacit knowledge through interactions between people, PC provides mechanisms for LM development. This output can be used by an extended group of people. PC comprises mechanisms of co-creation [7] which indicate first insights on how people create artifacts together with the help of collaborative technologies. The peers add value to the LM by yielding their own knowledge in form of learning content [7]. Until now, structure and learning objectives are open or predetermined by the lecturer [15], only sequences of a learning process are addressed, e.g. generating a short multiple-choice-task, and LM development is not reusable [7]. For developing LM [7] identified key principles as shown in table 1, which lead to first insights on how to design processes for documenting knowledge in a standardized and productive way. Nevertheless development of such learning processes often has a strong reference on specific content and context. So, reproducibility and assignability are difficult [16].

Table 1. Different kinds of peer learning [7], [11], [17],

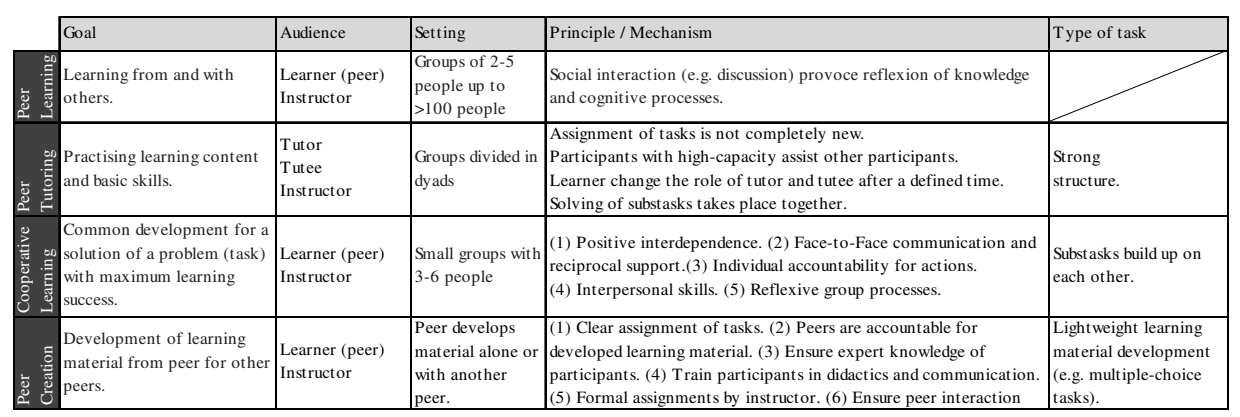

\subsection{Basics in Collaboration Engineering}

Knowledge documentation as a recurring task requires a structured and reusable collaborative process. Hence, CE research provides an approach for designing and conducting such processes to solve complex and recurring tasks. The more a task occurs, the more efficient it is to design it as a collaborative process and solve it with same process flow. Thereby a group of people works together towards a common goal while group activities are characterized by communication, cooperation and coordination $[18,19]$. These structured activities lead towards an additional benefit which cannot be attained by individual endeavor [3]. CE differentiates between three roles. A collaboration engineer designs and documents a collaborative process. A facilitator is able to design a non-recurring collaborative process. He disposes expert knowledge and moderation skills, so that he is able to conduct a process. A practitioner can act as facilitator or as participant of a collaborative process and is an expert on task and owns expert knowledge. The layer model of collaboration provides a framework for designing collaborative processes [20]. These layers are hierarchical and depend on each other: Goals as the first layer focus on defining a desired state or outcome as a group goal. The product layer addresses tangible or intangible artifacts as the outcome produced by a group. Defining and acquiring sub products in a collaborative process 
lead to one common product. Activities as the next layer describe particular subtasks a group must do to achieve defined products to fulfill the common goal. The subsequent layer addresses procedures. These are methods, strategies and tactics a group uses to execute work. So called patterns of collaboration - generate, reduce, clarify, organize, evaluate and build consensus - characterize how activities become structured and are observable regularities for the defined activities. The next layer contains tools and describes several technologies to support the execution of the collaborative process. Scripts as the last layer address documentation of behavior people say and do as they collaborate $[20,21]$. Thus, in context of knowledge documentation CE provides guidelines for designing a collaborative process with LM as collaborative product. Nevertheless educational claims are not anchored in CE so far.

\section{Framework for Developing a Peer-Creation-Process}

\subsection{Guiding Idea for a Peer-Creation-Process}

Based on the benefits of educational and CE we convey the idea of a PCP for documenting knowledge in form of LM. Thereto we deviate following assumptions starting with collaboration, the work of two or more people on common material, which is characterized by coordination, communication and cooperation [19]: (1) Collaboration enables exchange of heterogeneous knowledge; (2) Such exchange of heterogeneous knowledge provokes recapitulation of knowledge; (3) This in turn provokes a learning process for all involved people; (4) A learning process is the basis for correct documentation of knowledge from people at different levels of knowledge. (5) Documentation of knowledge comes to an end when quality of developed LM is high enough. Elsewise recapitulation, learning process and documentation are passed through again. Figure 1 depicts these assumptions.

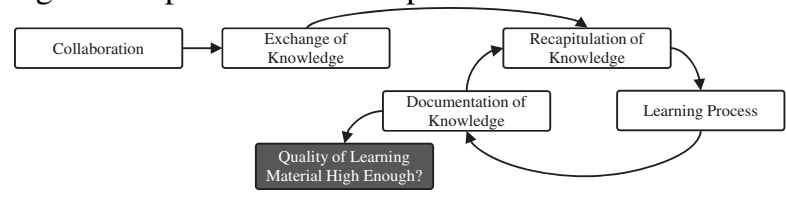

Fig.1. Research assumptions

Subsequently, collaboration is the basis for structuring a PCP. Thus, solutions become important which enable a collaborative exchange of knowledge while improving learning success of participants and documenting their expert knowledge and knowhow by generating LM. Hence, we use mechanisms from CE research enriched with educational research for designing a PCP for developing LM. Figure 2 demonstrates the intention of a PCP and points out the demand for two perspectives of analyses. (1) PCP for developing LM and (2) the distribution of developed LM used by an extended group of people. Focusing on the PCP, input is given by participants in form of peers at a different level of knowledge, so-called practitioners. A further input comes from a facilitator with moderation skills. The throughput addresses structured collaboration between practitioners guided by a facilitator. Though reciprocal interac- 
tions between practitioners take place which cause individual acquisition as well as transfer of knowledge and documentation of knowledge in a didactical reasonably way. This has two positive effects for the output of the PCP. Social interaction and the assignment of task in structuring knowledge in a didactical reasonable way provoke individual reflection of knowledge. Hence, high quality LM arises as main output whereas individual learning success by practitioners arises as a side effect. They are learning from and with each other during their collaboration. Thus, from an educational point of view, cognitive process dimensions like applying, analyzing, evaluating and creating knowledge [22] will be addressed. Focusing on the distribution of LM, an extended group of people can use it for improving individual learning success. It is expected that this kind of learning will address lower cognitive process dimensions like remembering or understanding [22].

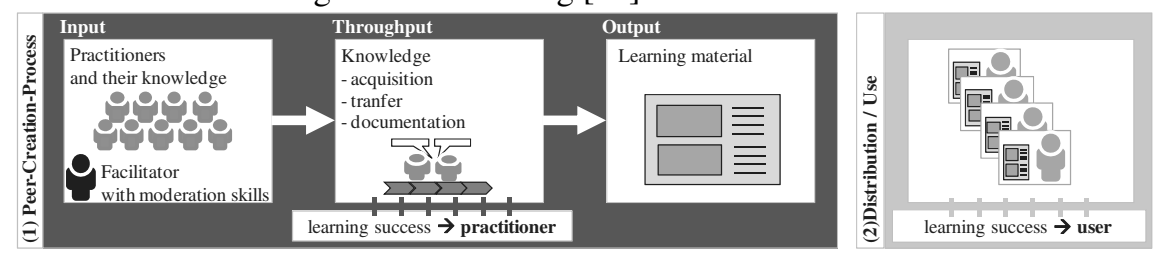

Fig. 2. Guiding idea for a peer-creation-process

\subsection{Requirements from Educational Research}

In order to design a PCP for developing LM some formal educational requirements need to be considered addressing PL and PC to mediate individual learning success and the development of LM. For that purpose we differentiate between the process and the output in form of LM. By focusing on the way the process has to be conducted, participants of the process should learn something. Through social interactions and collaboration with others they improve their own knowledge and document exchanged knowledge. Thus, knowledge creation, acquisition and transfer take place. This should be anchored in a didactical reasonable way, e.g. with useful learning objectives [22]. Besides that reciprocity in social interaction has to be provided between participants and direct feedback has to be conveyed to participants [14]. Further a lecturer should prepare basic conditions for peer learning activities, assist participants and communicate explicit expectations [14]. Continuing requirements arise from content of knowledge. Hence, the need for different process design depends on knowledge complexity. Therefore educational requirements influence the design of the PCP. By focusing on the output of PCP in form of LM, quality is necessary. This includes the following requirements. Conducted by the intention that an extended group of people can learn with this material didactical requirements like learning objectives [22] and structural requirements like coherent and logical content presentation should also be respected. This refers to the length and design. Further indicators of quality arise from the type of LM. So this could be a textual explanation, a learning exercise or an explanation video. In addition correctness of content should be ensured as a further indicator for LM quality [23]. Therefore controlling mechanisms like peer reviews can be a solution, which should be integrated into the design of PCP. 


\subsection{Requirements from Collaboration Engineering}

A central requirement from $\mathrm{CE}$ focuses on the success of a collaborative process with predictable and repeatable results. According to the layer model of collaboration [21] all layers will set requirements to the PCP, but several layers will become important from an educational point of view for expanding CE research. A common collaboration goal, which is congruent to individual goals of practitioners, should be anchored with clear learning objectives. So, a clear description of cognitive process dimension must be considered in process design [22]. In the context of product layer, tangible and intangible artifacts are existent like enhancing individual learning success by transfer, acquisition and documentation of knowledge through collaboration. Besides that, the outcome of PCP represents a tangible artifact in form of LM. However an intangible artifact in form of quality of LM has to be considered, too. Focusing on procedures, particularly on the patterns of collaboration and ThinkLets, we assume an integration of further educational requirements. To ensure LM quality direct feedback focusing on content of LM is needed. To ensure correctness of documented knowledge mechanisms like peer reviews have to be integrated. Several requirements are expected from collaboration scripts. All necessary expertise like moderation knowhow of a facilitator or pedagogical skills in form of hints for correct behavior in teaching situations, e.g. how to communicate feedback towards participants, should be contained in process design.

\subsection{Conceptual Framework}

In order to design a PCP for reusable development of LM we developed a framework in dependence on the established layer model of collaboration. Hence, figure 3 picks up the guiding idea of PCP, consolidates educational requirements for process design and highlights expected educational expansions in the layer model of collaboration.

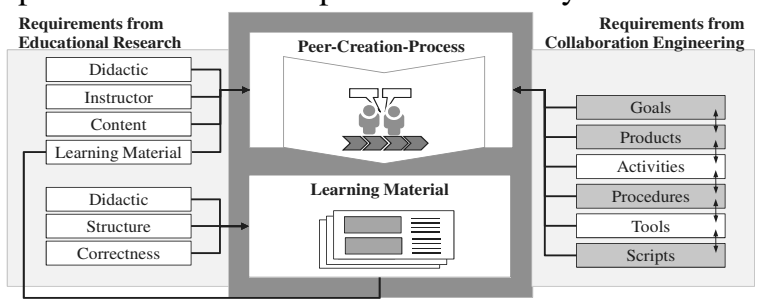

Fig. 3. Conceptual framework of integrating learning into group collaboration processes

The centre of figure 3 indicates the purpose of research with PCP and its output in form of LM. On the left, figure 3 visualizes educational requirements like didactic, instructor and content of knowledge influencing design of PCP with focus on how practitioners have to collaborate to achieve an individual learning success and work towards a common goal in form of LM. Besides that the figure visualizes the influence of LM type. This leads to deduction of quality requirements addressing didactic aspects, structure and correctness of LM in the design of PCP. These educational facts provide important inferences on the assignment of tasks respectively the way the col- 
laborative goal and product should be achieved. On the right requirements from $\mathrm{CE}$ are depicted and important layers with an expected educational influence are highlighted. In a structured process design requirements from educational research should be combined with CE especially the layers addressing goals, products, procedures and scripts. These are central elements for anchoring educational requirements, like peer reviews in the procedures or hints for pedagogical skills in the scripts.

\section{$4 \quad$ Next Steps and Expected Contribution}

As our next step, we currently plan a study addressing process design, learning success of participants and quality of LM in a large scale university lecture to evaluate our general idea. For that purpose we design and evaluate a PCP where participants develop a storyboard for an explanation video. Thus for our process design, we consider individual learning objectives in the goals layer. We consider intangible learning success as well as tangible LM in the product layer and ensure correctness of LM by integrating lightweight peer-reviews for several subtasks in procedures layer. In the scripts we integrate pedagogical techniques and advices. In the experimental setting we plan with about 300 participants which will randomly be assigned to the treatment (using the PCP) or control group. Within each group, we will randomly build another 6 groups so that roughly 25 students collaborate in each group. A quantitative inquiry of the participants will be conducted for gathering insights on process design and individual learning success. During a focus group qualitative interviews with experts in $\mathrm{CE}$ and education should display insights on the process design and the quality of developed LM. After a first successful evaluation of our approach with students, the PCP will be used by at least of our partners from industry, to develop LM supporting the knowledge transfer in the partner organization. In this paper we developed the vision of reusable PCP with the help of CE routines enriched with educational requirements. Therefore, we showed the concept of a PCP, and its potential for challenges in knowledge transfer and documentation. We identified several challenges for designing a PCP that require extension in the layer model of collaboration and conveyed this in a conceptual framework. Such processes have the potential to make organizations independent from educators and standardize inexplicit pedagogical methods and routines. Thus, it allows reusability and the execution by facilitators and practitioners with moderation skills. The output of the PCP can be used as knowledge base for acquisition of factual knowledge and thus, bring about a second opportunity for knowledge transfer towards an extended group of people. With the described research we expect extensions for the body of knowledge of collaboration. Further, the results provide first insights of a structured and reusable way for overcoming challenges in knowledge transfer and documentation.

\section{$5 \quad$ References}

1. Fuchs-Kittowski, F.: Interaktionsorientiertes Wissensmanagement. In: Krcmar, H. (ed.) Interaktionsorientiertes Wissensmanagement. Peter Lang GmbH, Frankfurt am Main (2013) 
2. Ries, B.C., Diestel, S., Shemla, M., Christina;, L.S., Jungmann, F., Wegge, J., Schmidt, K.H.: Age Diversity and Team Effectiveness. In: Schlick, C.M., Frieling, E., Wegge, J. (eds.) Age-Differentiated Work Systems. Springer, Berlin, Heidelberg (2013)

3. Bittner, E.A.C., Leimeister, J.M.: Creating Shared Understanding in Heterogeneous Work Groups: Why It Matters and How to Achieve It. JMIS, vol. 31, pp. 111-144 (2014)

4. Johnson, L., Adams Becker, S., Estrada, V., Freeman, A.: NMC Horizon Report: 2014 Higher Education Edition. The New York Media Consortium, Austin, Texas (2014)

5. Gregor, S., Hevner, A.R.: Positioning and Presenting Design Science Research for Maximum Impact. MIS Quarterly,vol. 37, pp. 337-355 (2013)

6. Gagne, R.M.: Learning Outcomes and Their Effects. American Psychologist, vol. 39, pp. 377-385 (1984)

7. Wegener, R., Leimeister, J.M.: Peer Creation of E-Learning Materials to EnhanceLearning Success and Satisfaction in an Information Systems Course. 20th ECIS, Barcelona (2012)

8. Dillenbourg, P.: What do you mean by collaborative learning. In: Dillenbourg, P. (ed.) Collaborative learning, pp. 1-19. Elsevier, Oxford (1999)

9. Arbaugh, J.B.: Online and Blended Business Education for the 21st Century: Current research and future directions. Woodhead Publishing (2010)

10. Damon, W.: Peer education. Jn. of Applied Developmental Psychology, pp.331-343(1984)

11. Topping, K.J.: Trends in Peer Learning. Educational Psychology, 25, pp. 631-645 (2005)

12. Geer, J., McCalla, G., Collins, J., Kumar, V., Meagher, P., Vassileva, J.: Supporting Peer Help and Collaboration in Distributed Workplace Environments. International Journal of Artificial Intelligence in Education, vol. 9, pp. 159-177 (1998)

13. Hua Liu, C., Matthews, R.: Vygotsky's philosophy: Constructivism and its critisms examined. International Education Journal, vol. 6, pp. 386-399 (2005)

14. Harris, A.: Effective Teaching. School Leadership \& Management, 18, pp. 169-183 (1998)

15. Auvinen, A.-M.: The challenge of quality in peer-produced eLearning content. eLearning Papers, vol. 17, pp. 1-11 (2009)

16. Kollar, I., Fischer, F., Hesse, F.W.: Collaboration Scripts - A Conceptual Analysis. Educational Psychology Review, vol. 18, pp. 159 - 185 (2006)

17. Büttner, G., Warwas, J., Adl-Amini, K.: Kooperatives Lernen und Peer Tutoring im inklusiven Unterricht. Zeitschrift für Inklusion, vol. 1-2 (2012)

18. Kolfschoten, G.L., Vreede, G.-J.: A Design Approach for Collaboration Processes: A Multimethod Design Science Study in Collaboration Engineering. JMIS, 26, 225-256 (2009)

19. Leimeister, J.M. (ed.): Collaboration Engineering - IT-gestützte Zusammenarbeitsprozesse systematisch entwickeln und durchführen. Springer Gabler, Berlin Heidelberg (2014)

20. Briggs, R.O., Kolfschoten, G.L., Vreede, G.-J., Albrecht, C., Dean, D., R.;, Lukosch, S.: A Seven-Layer Model of Collaboration. 30th ICIS, Phoenix, Arizona, USA (2009)

21. Briggs, R.O., Kolfschoten, G.L., Vreede, G.-J.d., Albrecht, C., Lukosch, S., Dean, D.L.: A Six-Layer Model of Collaboration. In: Jay F. Nunamaker Jr., Nicholas C. Romano Jr., Briggs, R.O. (eds.) Collaboration Systems, pp. 221-228. Advances in Management Information Systems. New York (2014)

22. Krathwohl, D.R.: A Revision of Bloom's Taxonomy: An Ovierview. Theory Into Practice, vol. 41, pp. 212-218 (2002)

23. Leacock, T.L., Nesbit, J.C.: A Framework for Evaluating the Quality of Multimedia Learning Resources. Educational Technology \& Society, vol. 10, pp. $44-59$ (2007) 